\title{
BMJ Open Rationale, design and baseline data of a mixed methods study examining the clinical impact of a brief transition programme for young people with juvenile idiopathic arthritis: the DON'T RETARD project
}

\author{
Deborah Hilderson, ${ }^{1,2}$ Rene Westhovens, ${ }^{3}$ Carine Wouters, ${ }^{4}$ Kristien Van der Elst,,${ }^{2,3}$ \\ Eva Goossens, ${ }^{2,5}$ Philip Moons ${ }^{2}$
}

To cite: Hilderson D, Westhovens R, Wouters C, et al. Rationale, design and baseline data

of a mixed methods study examining the clinical impact of a brief transition programme for young people with juvenile idiopathic arthritis: the DON'T RETARD project. BMJ Open 2013;3: e003591. doi:10.1136/ bmjopen-2013-003591

- Prepublication history for this paper is available online. To view these files please visit the journal online (http://dx.doi.org/10.1136/ bmjopen-2013-003591)

Received 15 July 2013 Revised 14 October 2013 Accepted 4 November 2013

CrossMark

For numbered affiliations see end of article.

Correspondence to Dr Philip Moons; philip. moons@med.kuleuven.be

\section{ABSTRACT}

Objectives: To describe (1) the content of a transition programme for young people with juvenile idiopathic arthritis (JIA) designed as a brief intervention, (2) the rationale and design of a mixed-methods study evaluating the clinical impact of this transition programme and (3) to provide baseline data of the intervention group.

Design: An 'embedded experimental' design is used for the evaluation of the transition programme. A 'onegroup pretest-posttest, with a non-equivalent posttestonly comparison group design' is used to quantitatively evaluate the impact of the transition programme, applying both longitudinal and comparative analyses. Subsequently, experiences of adolescents and their parents who participated in the experimental group will be analysed qualitatively using content analysis.

Setting: Participants in the intervention are recruited at a tertiary care centre in Belgium. The comparison group participants are recruited from one tertiary and three secondary care centres in Belgium.

Participants: The intervention group consists of 33 young people (25 females; 8 males) with a median age of 16 years. Main diagnoses are persistent or extended oligoarticular JIA (33\%), polyarticular JIA $(30 \%)$, enthesitis-related JIA $(21 \%)$ or systemic arthritis (15\%).

Intervention: The transition programme comprises eight key components: (1) transition coordinator; (2) providing information and education; (3) availability by telephone; (4) information about and contact with an adult care programme; (5) guidance of parents; (6) meeting with peers; (7) transfer plan; and (8) actual transfer to adult care.

Primary and secondary outcomes: The primary outcome is health status, as perceived by the adolescents. Secondary outcomes are health status, as perceived by the parents; medication adherence; illness-related knowledge; quality of life; fatigue; promotion of independence; support of autonomy; behavioural control and psychological control.
Strengths and limitations of this study

- A transition programme for adolescents with juvenile idiopathic arthritis is developed as a brief intervention, which may be less costly and time-consuming than the existing, more comprehensive transitional care interventions.

- The development and evaluation of this transition programme is guided by the initial Medical Research Council framework for complex interventions.

- The use of a sequential mixed methods approach within the framework for complex intervention enables a full and integrative insight into the clinical impact of the key components of the transition programme.

Results: At baseline, the median score was 69.2 $(Q 1=60.0 ; Q 3=92.9)$ on psychosocial health and 68.8 $(Q 1=56.3 ; Q 3=89.1)$ on physical health. Rheumaticspecific health scores ranged from 62.5 to 100 .

Conclusions: We present the rationale and design of a study intended to evaluate a transition programme for adolescents with JIA as a brief intervention.

\section{BACKGROUND}

Some decades ago, several paediatric disorders were associated with high, progressive morbidity and increased mortality. For many people suffering from these chronic diseases, medical, surgical and technological advancements have resulted in improved disease management and increased life expectancy. Unlike in the past decades, children with a progressive deteriorating disorder now often live into adulthood managing a chronic disease. Expert lifetime care should be 
provided for these patients in order to maximise their potential and lifelong functioning.

Young people with chronic conditions undergo different stages during their life. Two of the many important phenomena that occur include a developmental transition into adulthood, a phase during which young people evolve from being a dependent child to becoming an independent adult. ${ }^{1}$ Second, their setting of care is transferring from a paediatric context to an adultfocused environment. Indeed, a timely and wellprepared transfer to adult-centred care is advocated. ${ }^{2}{ }^{3}$ This transfer is defined as an event or series of events through which adolescents and young adults with chronic physical and medical conditions move their care from a paediatric to an adult healthcare environment. ${ }^{4}$ According to the recent literature, the paediatric-toadult transfer of care should be preceded by a preparatory transitional phase. Transition is therefore defined as a process by which adolescents and young adults with chronic childhood illnesses are prepared to take charge of their lives and their health in adulthood. ${ }^{4}$

In order to prepare young people to take on new responsibilities for their health and to anticipate the imminent transfer to adult care, transition programmes have been developed. The efficacy of these transition programmes has been evaluated to some extent in the last decade. $^{5-11}$ Using quasi-experimental designs, investigators have found some positive effects on the quality of life, ${ }^{8}$ disease outcomes, ${ }^{57}$ number of admissions and the length of stay of readmissions, ${ }^{7}$ knowledge, ${ }^{8}$ work experience and career advice ${ }^{8}$ and satisfaction with care. ${ }^{10}$ The vast majority of the studies have been conducted in the $\mathrm{UK},{ }^{12}$ which may limit the generalisability of the study findings to other healthcare systems due to, for example, differences in financing and reimbursement of healthcare expenditures. Therefore, research on the efficacy of transition programmes in other countries is warranted. ${ }^{12}$

Existing transition programmes generally adopt a comprehensive approach that is very likely contributing to their positive effects. However, such extensive programmes are also perceived as being costly and timeconsuming to implement in day-to-day practice, although economic cost-effectiveness studies are currently lacking. This is illustrated by the finding that the most frequently reported barriers to the formal transition of patients are limited time and lack of funding for a transition coordinator. ${ }^{13}{ }^{14}$ Hence, the costeffectiveness of transition programmes can be questioned. In times of economic crisis and limited funding, more sustainable alternatives must be explored. Brief evidence-based interventions have the potential to be cost-effective and therefore are more likely to be implemented in clinical practice.

Transition programmes can be viewed as complex interventions. Complex interventions are built up from a number of components that may act independently and interdependently. ${ }^{15}$ This implies that the active ingredient of the intervention is unknown or difficult to specify.
The British Medical Research Council (MRC) has provided a framework for developing and evaluating complex interventions. It entails a recursive process of development, feasibility and pilot testing, evaluation and implementation of the complex intervention. Hence, before any formal efficacy assessment can be performed, comprehensive preparatory work is conducted. ${ }^{15}$

The original model of the MRC comprised an investigative sequence of five phases (figure 1). First, theory and evidence are assessed in order to provisionally identify the steps and the key components of the intervention; this is termed the preclinical phase. Second, an understanding of the intervention and its possible effects is developed; this is termed phase I: the modelling phase. Third, the feasibility of key components is assessed, and the recruitment procedures and measurements of outcomes are tested; this is termed phase II: the exploratory trial phase. Fourth, randomised controlled trials are conducted to evaluate the impact of the complex intervention. These trials require adequate power, adequate randomisation, appropriate outcome measures and other standard features of well-designed trials; this is termed phase III: the definitive randomised controlled trial. Finally, separate studies are conducted to establish the long-term and real-life effectiveness of the intervention; this is termed phase IV: the long-term implementation. ${ }^{15} 16$

In order to develop and test a transition programme involving a complex and brief intervention, we established the DON'T RETARD project (Devices for the OptimisatioN of TRansfEr and Transition of Adolescents with Rheumatic Disorders). This project was designed for young people with juvenile idiopathic arthritis (JIA) (http://www.kuleuven.be/switch2/rheuma.html) and aimed to provide a proof of concept for a transition programme devised as a brief intervention. We followed the original MRC framework for complex interventions. As part of the modelling phase, we conducted some preparatory studies that were previously published elsewhere. ${ }^{14} 17$ The next step in the DON'T RETARD project is to evaluate the newly developed transition programme.

In the present article, we aim (1) to extensively describe the content of a transition programme for young people with JIA that was designed as a brief intervention, (2) to describe the rationale and design of a mixed methods study evaluating the clinical impact of this transition programme and (3) to report the baseline characteristics of the intervention group on the respective primary and secondary outcomes. We hypothesise that the transition programme would improve the physical, psychosocial and rheumatic-specific health of adolescents with JIA (primary outcome). ${ }^{8}{ }^{18}$ Second, we hypothesise that the programme would improve medication adherence, illness-related knowledge, quality of life, threshold to fatigue and parenting styles in patients who participate in the programme (secondary outcomes). To guarantee the transparency and quality of describing this complex intervention, we used the recently 
Figure 1 Development of a complex intervention based on the Medical Research Council framework and its evaluation using an embedded experimental design.

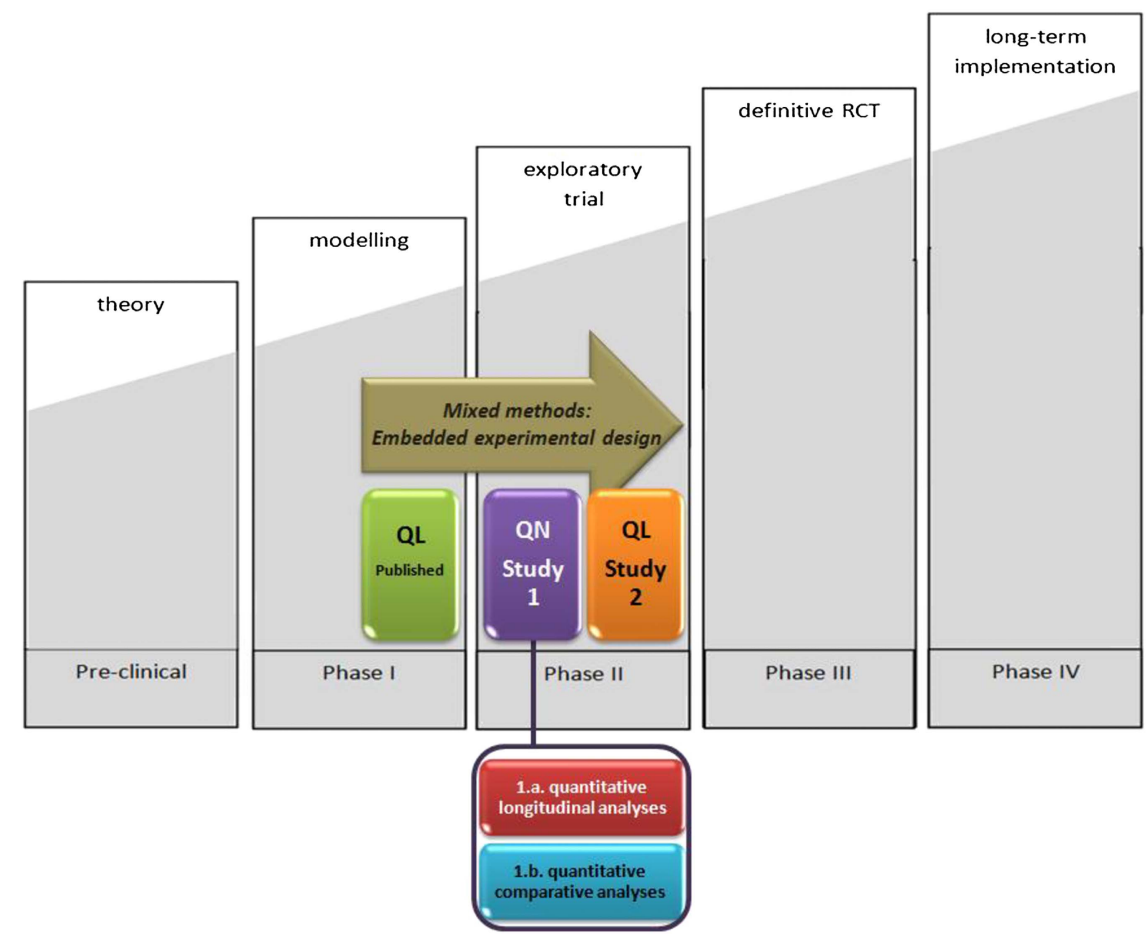

published criteria for reporting the development and evaluation of complex interventions (CReDECI). ${ }^{19}$ The results on the clinical impact of the transition programme will be reported in a forthcoming publication.

\section{Transition programme as a brief intervention}

Our transition programme, which is designed to be a brief intervention, contains five steps. We will describe each step in the next section.

\section{Intervention steps}

1. The first step occurs during a scheduled outpatient visit. The paediatric rheumatologist introduces the transition coordinator (TC) to the patient (aged 1416 years) and his or her parents, and explains the transition programme. During this first face-to-face visit with the $\mathrm{TC}$, the $\mathrm{TC}$ provides the patient and parents with a rheumatology management diary (ie, a booklet including self-reporting symptoms scales, an overview of scheduled appointments, space for writing down questions, etc), written information about JIA and medication management and a DVD with instructions regarding appropriate exercising. The TC guides the young person and parent(s) through three patient information websites and presents a video about JIA and its consequences. Young people have the possibility to see the rheumatologist and TC without the presence of their parent(s). This independent visit is strongly encouraged. Furthermore, a second face-to-face visit with TC is scheduled. The initial contact session lasts about 30$40 \mathrm{~min}$. The TC is, however, continuously available by telephone to answer additional questions about the condition, therapy, transition process, health behaviour and clinic appointments.

2. The second step of the intervention occurs 6 months later, and consists of a second face-to-face visit with TC, taking place at the paediatric outpatient clinic. During this visit, TC discusses health behaviour and deals with fatigue, school, friends, self-image, knowledge about the disease and any difficulties with medication adherence. Participants receive a folder about the practical issues related to the adult rheumatology programme (eg, contact information for the secretary and rheumatologists, organisational information for the outpatient clinic). This session lasts approximately $30 \mathrm{~min}$.

3. For the third step of the intervention, patients and their parents are invited to attend an adolescent information day. On this day, all patients who attended their second visit with the TC are invited to come together. The adolescent information day has two parallel programmes: one for the adolescents and one for the parents. The programme for adolescents begins with a brief introduction from the TC and the paediatric rheumatologist. Then the adolescents take part in a variety of activities: they meet with peers, take an orientation tour at the adult care facilities, meet with the rheumatologists, physiotherapist(s) and nurse specialist of the adult rheumatology team, and attend a workshop on psychological issues. These activities are followed by a cooking workshop, in which the adolescents prepare a complete meal for themselves and their parents. This allows them to talk with their peers in an informal setting. 
4. The parallel programme for parents during this third step of the intervention is similar. A parent association representative is available so that the parents can express their concerns about their child's transition to adulthood. Activities include taking an orientation tour at the adult care facilities, meeting with the rheumatologists and nurse specialist of the adult rheumatology team, and attending a lecture on psychological issues inherent to the development of an adolescent with chronic disorders. After the formal programme, parents join their children for the dinner prepared by the children. The adolescent information day starts at 14:00 and ends at 18:30, and takes place twice during the transition programme. For each information day, about 15 patient-parents dyads participate.

5. For the fourth step, an individualised transfer plan is developed by the TC based on conversations with the young person and the parents. This plan is developed before transferring the patient from paediatric to adult rheumatology. This plan is based on the coding structure of the International Classification of Functioning, Disability and Health for Children and Youth (ICF-CY). By shifting the focus from cause to impact, the ICF-CY puts all health conditions on an equal footing, allowing them to be compared using a common metric. Furthermore, it takes into account the social aspects of a disability and does not view disability only as a 'medical' or 'biological' dysfunction. By including contextual factors, in which environmental factors are listed, the ICF-CY allows us to record the impact of an environment on a person's functioning. The transfer plan is also based on patient information collected by the paediatric rheumatologist, the TC and other healthcare professionals that are consulted. The development of a transfer plan requires $30 \mathrm{~min} /$ patient.

6. The fifth step is the actual transfer. Once the transfer plan is handed over to the adult rheumatologist, the patient is considered transferred to an adult rheumatology care. The third face-to-face visit with the TC occurs during the patient's first outpatient visit to the adult rheumatology care. This time, the rheumatologist of the adult rheumatology programme joins the session, along with the TC, patient and parents. The TC focuses on health behaviour and medication information. During this outpatient visit, the patient is formally 'handed over' to the adult rheumatology providers. This session lasts about $20 \mathrm{~min}$.

\section{Intervention key components}

The transition programme comprises eight key components that are implemented in one or more of the five steps: (1) a transition coordinator; (2) providing information and education about JIA and medication management, health behaviour, dealing with fatigue, school, friends and any problems with medication adherence; (3) availability by telephone; (4) information about and contact with the adult rheumatology programme;
(5) guidance of parents; (6) meeting with peers; (7) a transfer plan and (8) the actual transfer to the adult rheumatology programme.

Altogether, TC spends 60-90 min/patient, spread over a period of 1.5 years. This is in addition to the 40 manhours (for the TC, paediatric rheumatologists, rheumatologists of the adult setting, physiotherapists, nurses of adult rheumatology, psychologists) incurred by the adolescent information day activities and the $20 \mathrm{~h}$ needed for its preparation. Overall, it was estimated that the time and work investment of one full-time equivalent is needed to implement this brief intervention for a caseload of 250-300 transitioning patients.

\section{METHODS AND DESIGN}

To develop and evaluate the transition programme, we apply a mixed methods approach in which quantitative and qualitative studies are combined. More specifically, we use an 'embedded experimental design'. ${ }^{20}$ This design is characterised by a set of qualitative studies that are conducted before and after a quantitative study. In our project, the embedded experimental design starts with a qualitative study using in-depth interviews. The aim of these interviews is to better understand the transitional needs of young people with JIA. The information obtained in this qualitative study assisted us in developing the transition programme. Hence, this particular qualitative study corresponds to the modelling phase of the MRC framework (figure 1, green box). The methods and results of this first qualitative study are reported in two related articles. ${ }^{21} 22$ Therefore, we do not elaborate on this study in the present article. However, we did not rely solely on our qualitative findings to determine the content of the transition programme. Indeed, we built on previously published studies on transitional care. ${ }^{8} 10$ 21-23

Subsequently, we conduct a quasi-experimental study employing a 'one-group pretest-posttest, with a non-equivalent posttest-only comparison group' design (figure 1, purple box; figure 2). In this quantitative study, we investigate the clinical outcomes of patients with JIA and their parents who participate in the transition programme. In this respect, longitudinal analyses will be conducted to investigate changes over time (figures 1 and 2; indicated in red). Furthermore, comparative analyses will be performed by comparing the post-test scores of the intervention group with those of the patients who received usual care (figures 1 and 2; indicated in blue). This quantitative study is called study 1 in figure 2. After this initial quantitative study, a second-qualitative study using an explanatory design is conducted (study 2; indicated in orange), in which we elaborate on the experiences of adolescents with JIA and their parents regarding their participation in the transition programme. Both studies 1 and 2 are considered to correspond to the exploratory trial phase of the MRC framework (see Background section). 
Figure 2 Flow chart illustrating the quantitative and qualitative studies used to assess the transition programme for adolescents with juvenile idiopathic arthritis. In study 1 , quantitative analyses were conducted based on a one-group pretest-post-test design with a non-equivalent posttest-only comparison group composed of adolescent-parent dyads. This was followed by study 2 , a qualitative study consisting of in-depth interviews.

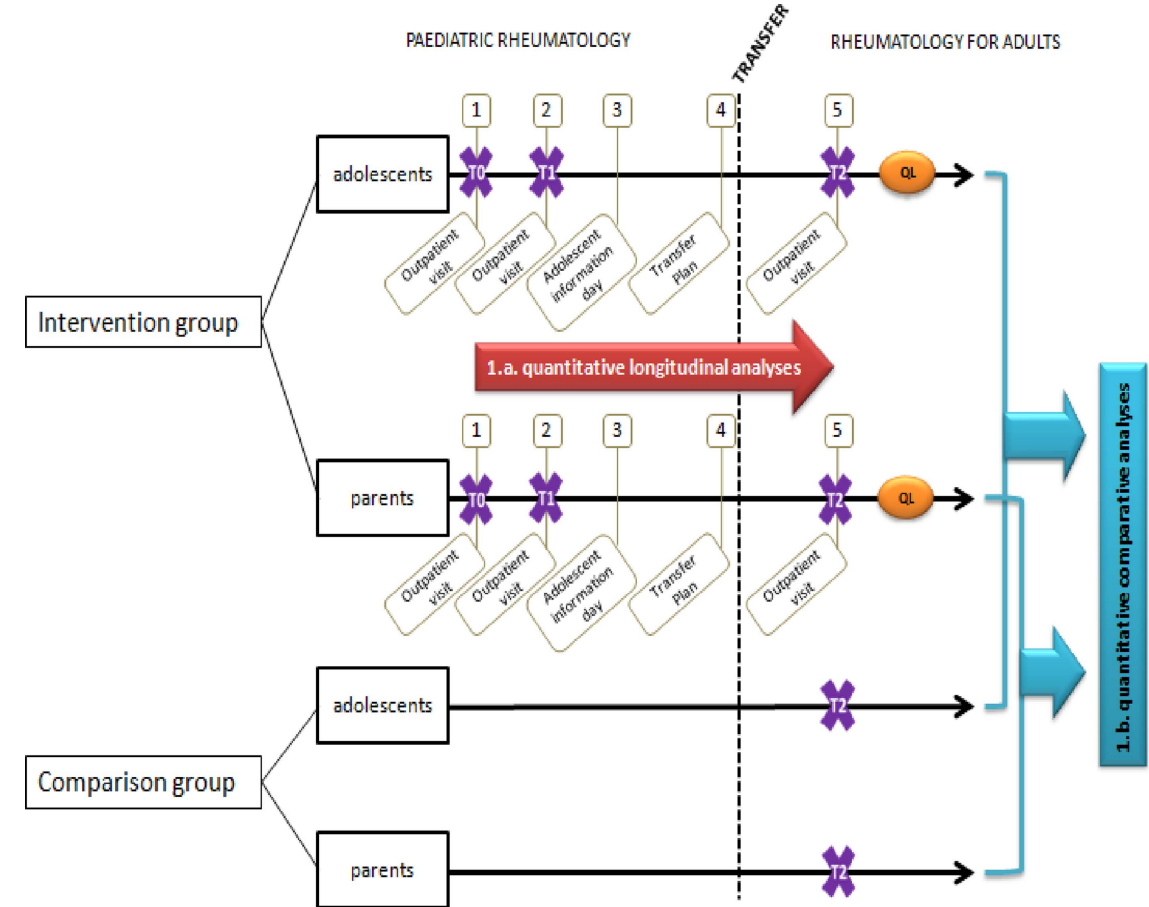

Legend: $\$$ quantitative measurements $\square$ intervention component
Study 1.a. Quantitative study: Iongitudinal analysis Inclusion criteria

Potential participants are recruited during a scheduled visit at the outpatient clinic, between 1 February 2009 and 1 February 2011. Dutch-speaking young people (14-16 years of age) with JIA, treated and in active follow-up at the Department of Paediatric Rheumatology of the University Hospitals Leuven, are invited to participate in the intervention trial. Patients are excluded if they have developmental or cognitive disabilities or if they do not have the physical capacity to complete the questionnaires used in the study. The convenience sample includes all eligible participants.

\section{Informed consent}

Patients are included after obtaining written informed consent from the parents and informed assent from the patients (all patients were minors at the time of inclusion). Anonymity is guaranteed, and patients and parents are assured that they could stop participating at any time.

\section{Variables and measurements}

All participants are assessed three times: at baseline $\left(\mathrm{T}_{0}\right)$, at the second outpatient visit at paediatric rheumatology $\left(\mathrm{T}_{1}\right)$, and at the first outpatient rheumatology consultation in the adult care setting ( $\mathrm{T}_{2}$; figure 2). At each point in time, we measure the primary and secondary outcomes as well as the disease parameters. The primary outcome of interest is the self-perceived health status of the patient, as measured with the Pediatric Quality of Life Inventory (PedsQL $\left.{ }^{\mathrm{TM}}\right)$. We use the generic (PedsQL 4.0 Generic Core Scales) and the disease-specific module (PedsQL 3.0 Rheumatology Module). ${ }^{24-26}$

We measure the following set of secondary outcomes salient to the young people: the patients' health status as perceived by their parents, which is measured with the PedsQL 4.0 Generic Core Scales and PedsQL 3.0 Rheumatology Module. ${ }^{24-26}$ Furthermore, a set of patients' self-reported secondary outcomes are assessed: medication adherence is measured using a Visual Analogue Scale (VAS) and the Swiss HIV Cohort Study Adherence Questionnaire (SHCS-AQ) ${ }^{27}$; illness-related knowledge is measured using the modified Patient Knowledge Questionnaire (PKQ) ${ }^{28}$; and the level of global quality of life is measured using a Linear Analogue Scale (LAS). ${ }^{29}$ Fatigue is measured using the Multidimensional Fatigue Inventory (MFI-20) ${ }^{30}$ Finally, in the group of parents, four dimensions of parenting are assessed using parent-reported instruments. Promotion of independence by parents is measured using the Promotion of Independence Scale ${ }^{31}$ and support of autonomy is measured using the Autonomy Support Scale ${ }^{32}$ Furthermore, the level of behavioural control (ie, parental monitoring of child's behaviour) is assessed using the Parental Regulation Scale, ${ }^{33}$ while the aspect of psychological control (ie, intrusive and manipulative form of controlling) is measured using the Psychological Control Scale. ${ }^{33}$ These outcomes are all found to be suboptimal in patients with JIA. ${ }^{23} 31{ }^{33-35}$ We hypothesise that participation in the transition programme could improve medication adherence, illness-related knowledge, quality of life, fatigue and parenting style. 
With regard to the disease parameters, we evaluate the clinical status of the disease activity and clinical remission on/off medication according to the preliminary criteria of Wallace et al. ${ }^{36}$ Furthermore, the functional status is assessed by the patient-reported and parent-reported Child Health Assessment Questionnaire (CHAQ-DI) ${ }^{37-39}$ Detailed information about the instruments used to assess the clinical impact of the transition programme are shown in table 1 .

\section{Data analysis}

Since this study is an exploratory trial formulated according to the MRC framework of complex interventions, our aim is not to test the efficacy of the transition programme; rather, it is a 'proof of concept' for the transition programme as a brief intervention. Therefore, we will not look for statistical significances but rather for clinical differences. Differences in patient-related and parent-related outcomes, which are measured continuously starting at $T_{0}$ (before intervention) to $T_{2}$ (after intervention), will be expressed as effect sizes.

For continuous variables, an effect size for the Wilcoxon test will be calculated by using $r=Z / v n$, where $\mathrm{Z}$ is the normal approximation of the Wilcoxon test statistic. To appraise the magnitude of the effect sizes, we will use Cohen's r. An effect size of $0.1-0.3$ is considered to be small, whereas effect sizes of $0.3-0.5$ and $\geq 0.5$ are considered to be medium and large, respectively. ${ }^{40}$

Adherence will be dichotomised as adherent or nonadherent. For this outcome, ORs will be calculated to report the effect size. The OR is the ratio of the odds of an outcome (eg, being adherent) of one group ( $\mathrm{T}_{0}$, before intervention) to the odds of the outcome of the other group $\left(T_{2}\right.$, after intervention). To appraise the magnitude of this OR, we will use the following suggested cut-off values: $1.5-2.5$ is a small effect; $2.5-4.3$ is a medium effect; and 4.3 or higher is a large effect. ${ }^{41}{ }^{42}$

Nominal data will be expressed in frequencies and percentages. Medians and quartiles (Q1-Q3) will be calculated for continuous, non-normally distributed variables.

\section{Baseline data}

The intervention group consists of 33 young people, 25 of whom are female and 8 male. Detailed information about JIA subtype, prescribed medication and remission status (on or off therapy) and the outcome data is shown in table 2. At baseline, patients reported a median score on psychosocial health of $69.2(\mathrm{Q} 1=60.0$; $\mathrm{Q} 3=92.9)$ and $68.8(\mathrm{Q} 1=56.3 ; \quad \mathrm{Q} 3=89.1)$ on physical health (PedsQoL). For rheumatic-specific health (PedsQoL), median scores ranged between 62.5 and 100 , with the worst scores for pain and hurt and the best scores for daily activities (PedsQoL). Furthermore, patients reported a median score for functional status (CHAQ-DI) of 0.3 (Q1=0.1; Q3=0.6) and 73.0 (68.5; 90.0) on the quality of life (LAS). Regarding fatigue, the scores on the motivation subscale were worst (7.0).

\section{Ethical issues}

The Institutional Review Board of the University Hospitals Leuven, Belgium evaluated and approved the study protocol (B32220096363). This study is performed with ethical standards as described in the latest Declaration of Helsinki.

\section{Study 1.b: Quantitative study: comparative analysis Inclusion criteria}

The intervention group's participant inclusion criteria are described above. The comparison group consist of 45 young people aged 17-23 years and their parents. These patients already have been transferred to the adult rheumatology programme without participating in a specific self-management/transition programme (ie, they received the usual care). The comparison group participants were recruited from four different health centres in Belgium. Patients who have developmental or cognitive disabilities or do not have the physical capacity to complete the questionnaires are excluded. Participants of the comparison group $(n=23)$ are included in the analyses if they can be matched with an intervention group participant in terms of disease activity, medication prescribed, JIA subtype and gender.

\section{Definition of usual care}

For the purposes of this study, usual care is defined as the care that is currently provided in day-to-day clinical practice. Young people with JIA are usually followed up at the paediatric rheumatology programme until the age of 16 years. Around that age, the adolescent might have a first outpatient visit with the adult rheumatologist and the paediatric rheumatologist together. When the rheumatologists, the patient and his or her parents agree that the patient is ready for transfer, an appointment for an adult rheumatology consultation is made. No TC or a formal transition plan is provided to patients who receive the usual care.

\section{Informed consent from the comparison group subjects}

We use the same procedure described above that we use for the intervention group; the young people above 18 years of age provide their own written informed consent.

\section{Variables and measurements}

The variables and measurements used in these comparative analyses are the same as those used in the longitudinal analyses (see above). The measures for the comparison group are acquired during one of the scheduled outpatient visits at the adult care clinic $\left(\mathrm{T}_{2}\right.$; see figure 2).

\section{Data analysis}

As with the longitudinal analyses, we are interested in clinical differences instead of statistical differences. In the comparative analyses, the scores of the intervention and comparison groups at $\mathrm{T}_{2}$ will be compared. The 
Table 1 Overview of variables and measurements in the quantitative study

\begin{tabular}{|c|c|c|c|c|c|c|c|}
\hline Variable & Measurement & Report & Items & Validity & Reliability & Responsiveness & Interpretation \\
\hline \multirow[t]{2}{*}{$\begin{array}{l}\text { Perceived health } \\
\text { status }\end{array}$} & $\begin{array}{l}\text { Paediatric Quality of Life } \\
\text { Inventory (PedsQL } 4.0 \text { ) } \\
\text { Generic Core Scale }\end{array}$ & $\begin{array}{l}\text { PAT \& } \\
\text { PAR }\end{array}$ & 23 & $\begin{array}{l}\text { Construct validity } \\
\text { confirmed, } \\
\text { ref. } 24, \text { p.333-335; } \\
\text { ref. } 25, \text { p.809; } \\
\text { ref. } 26, \text { p.719 }\end{array}$ & $\begin{array}{l}\text { Internal consistency confirmed } \\
\text { Adolescents' self-report: } \\
\text { Total score, } \alpha=0.91-0.92 ; \\
\text { physical health, } \alpha=0.83-0.90 ; \\
\text { psychosocial health, } \\
\alpha=0.87-0.89 \\
\text { Parents' proxy report } \\
\text { Total score, } \alpha=0.92-0.94 ; \\
\text { physical health, } \alpha=0.88 ; \\
\text { psychosocial health, } \alpha=0.89- \\
0.91,{ }^{24} \text { p.335;, }{ }^{26} \text { p.718 }\end{array}$ & $\begin{array}{l}\text { Responsiveness } \\
\text { confirmed, }^{26} \\
\text { p.721-722 }\end{array}$ & $\begin{array}{l}\text { Scores from } 0 \text { to } 100 \\
\text { Higher scores indicate a } \\
\text { better perceived health } \\
\text { status }\end{array}$ \\
\hline & $\begin{array}{l}\text { Paediatric Quality of Life } \\
\text { Inventory (PedsQL 3.0) } \\
\text { Rheumatology Module }\end{array}$ & $\begin{array}{l}\text { PAT \& } \\
\text { PAR }\end{array}$ & 22 & $\begin{array}{l}\text { Construct validity } \\
\text { confirmed, }^{26} \\
\text { p. } 720-721\end{array}$ & $\begin{array}{l}\text { Internal consistency confirmed } \\
\text { Adolescents' self-report } \\
\text { pain and hurt, } \alpha=0.90 \text {; daily } \\
\text { activities, } \alpha=0.84 \text {; treatment, } \\
\alpha=0.77 ; \text { worry, } \alpha=0.81 ; \\
\text { communication, } \alpha=0.79 \\
\text { Parents' proxy report : } \\
\text { Pain and hurt, } \alpha=0.90 \text {; daily } \\
\text { activities, } \alpha=0.89 \text {; treatment, } \\
\alpha=0.77 ; \text { worry, } \alpha=0.80 ; \\
\text { communication, } \\
\alpha=0.91,{ }^{26} \text { p. } 718\end{array}$ & $\begin{array}{l}\text { Responsiveness } \\
\text { confirmed }\end{array}$ & $\begin{array}{l}\text { Scores from } 0 \text { to } 100 \\
\text { Higher scores indicate a } \\
\text { better perceived } \\
\text { rheumatological health } \\
\text { status }\end{array}$ \\
\hline \multirow[t]{2}{*}{$\begin{array}{l}\text { Medication } \\
\text { adherence }\end{array}$} & $\begin{array}{l}\text { Visual Analogue Scale } \\
\text { (VAS) }\end{array}$ & PAT & 1 & NR & NR & NR & $\begin{array}{l}\text { Scores from } 0 \text { to } 100 \\
\text { Higher scores indicate } \\
\text { better medication } \\
\text { adherence }\end{array}$ \\
\hline & $\begin{array}{l}\text { SWISS HIV Cohort } \\
\text { Study Adherence } \\
\text { Questionnaire } \\
\text { SHCS-AQ }^{27}\end{array}$ & PAT & 2 & NR & NR & NR & $\begin{array}{l}\text { medication adherent or } \\
\text { non-adherent }\end{array}$ \\
\hline $\begin{array}{l}\text { Illness-related } \\
\text { knowledge }\end{array}$ & $\begin{array}{l}\text { The Modified Patient } \\
\text { Knowledge } \\
\text { Questionnaire (PKQ) }\end{array}$ & PAT & 16 & $\begin{array}{l}\text { Content validity } \\
\text { confirmed }^{28}\end{array}$ & NR & NR & $\begin{array}{l}\text { Scores from } 0 \text { to } 100 \\
\text { Higher scores indicate } \\
\text { more illness-related } \\
\text { knowledge }\end{array}$ \\
\hline Global quality of life & $\begin{array}{l}\text { Linear Analogue Scale } \\
\text { (LAS) }\end{array}$ & PAT & 1 & $\begin{array}{l}\text { Content validity } \\
\text { confirmed; } \\
\text { construct validity } \\
\text { confirmed, }{ }^{29} \text { p. } 410\end{array}$ & $\begin{array}{l}\text { stability confirmed }(I C C=0.65 \\
p<0.001),{ }^{29} \text { p.410 }\end{array}$ & $\begin{array}{l}\text { Responsiveness } \\
\text { confirmed, }{ }^{29} \text { p.410 }\end{array}$ & $\begin{array}{l}\text { Scores from } 0 \text { (worst } \\
\text { imaginable quality of life) } \\
\text { to } 100 \text { (best imaginable } \\
\text { quality of life) }\end{array}$ \\
\hline Fatigue & $\begin{array}{l}\text { Multidimensional } \\
\text { Fatigue Inventory } \\
\text { (MFI-20) }\end{array}$ & PAT & 20 & $\begin{array}{l}\text { Construct validity } \\
\text { confirmed, }{ }^{30} \text { p. } 6\end{array}$ & $\begin{array}{l}\text { Internal consistency confirmed: } \\
\text { General fatigue, } \alpha=0.82 \\
\text { physical fatigue, } \alpha=0.81\end{array}$ & $\begin{array}{l}\text { Responsiveness } \\
\text { confirmed, }^{30} \text { p. } 6\end{array}$ & $\begin{array}{l}\text { Scores from } 4 \text { to } 20 \\
\text { Higher scores indicate a } \\
\text { higher degree of fatigue }\end{array}$ \\
\hline
\end{tabular}

Inventory (PedsQL 3.0)

PAT \&

confirmed, ${ }^{26}$

p. $720-721$

physical fatigue, $\alpha=0.81$ 
Table 1 Continued

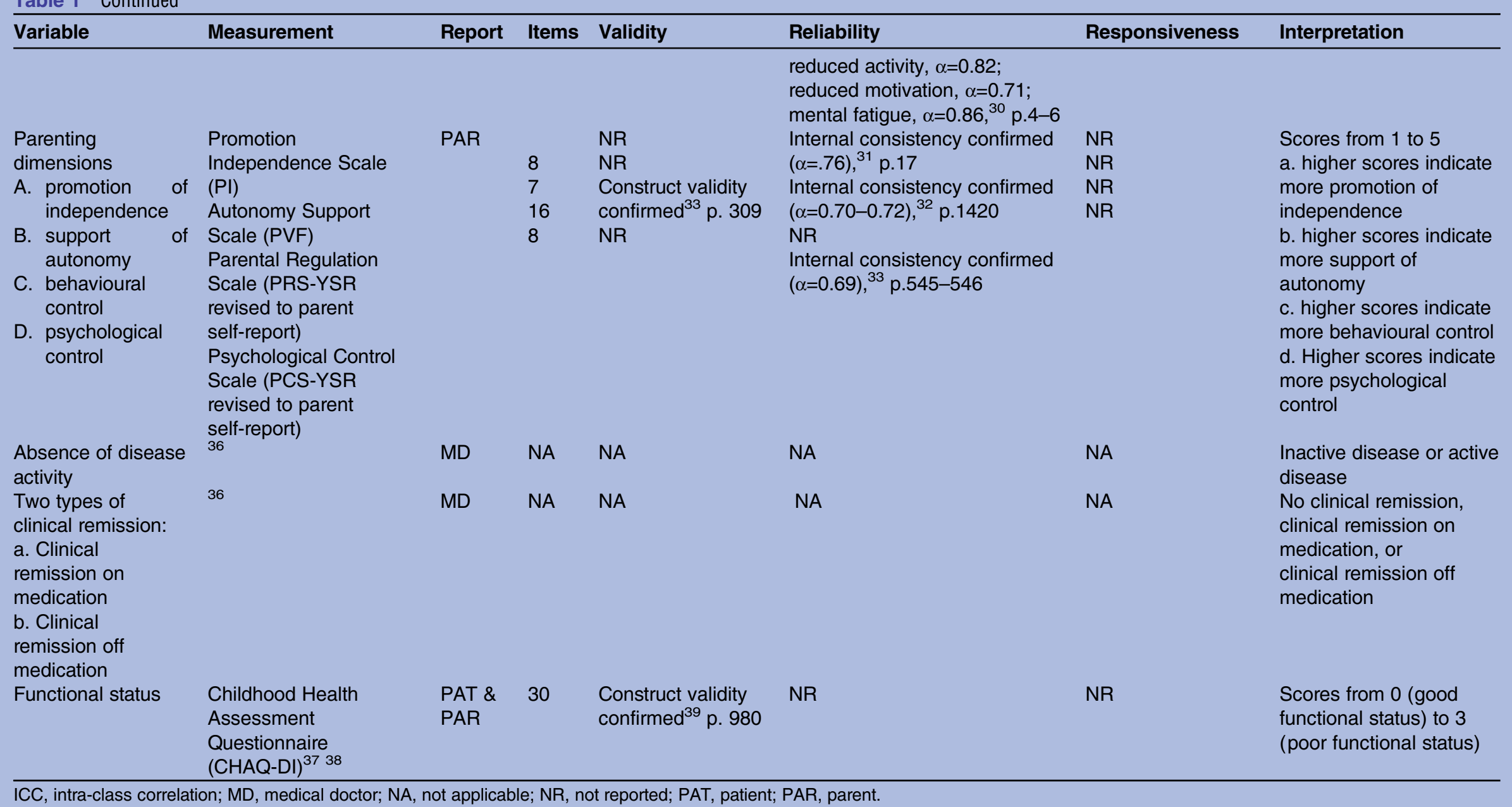


Table 2 Demographic, clinical characteristics and baseline outcome data of young people with JIA included in the intervention group $(n=33)$

\begin{tabular}{|c|c|}
\hline Baseline data & \\
\hline \multicolumn{2}{|l|}{$\operatorname{Sex} n(\%)$} \\
\hline Female & $25(75.8)$ \\
\hline Male & $8(24.2)$ \\
\hline \multicolumn{2}{|l|}{ Subtype of JIA based on preliminary criteria of Wallace ${ }^{36} n(\%)$} \\
\hline Persistent/extended oligoarticular JIA & $11(33.3)$ \\
\hline Polyarticular JIA (RF-, RF+) & $10(30.3)$ \\
\hline Systemic arthritis & $5(15.1)$ \\
\hline Enthesitis-related arthritis & $7(21.2)$ \\
\hline Age median, in years $\left(Q_{1} ; Q_{3}\right)$ & $16(15.2 ; 17.1)$ \\
\hline Presence of JIA disease activity $n(\%)$ & $10(30.3)$ \\
\hline Functional status (CHAQ-DI patient report) median $\left(Q_{1} ; Q_{3}\right)$ & $0.3(0.1 ; 0.6)$ \\
\hline Presence of clinical remission on therapy $n(\%)$ & $17(51.5)$ \\
\hline Presence of clinical remission off therapy $n(\%)$ & $5(15.2)$ \\
\hline Prescribed medication $\mathrm{n}(\%)$ & $23(69.7)$ \\
\hline NSAIDS & $14(60.9)$ \\
\hline DMARDS & $10(43.5)$ \\
\hline Biologicals & $6(26.0)$ \\
\hline Glucocorticoids & $4(17.4)$ \\
\hline \multicolumn{2}{|l|}{ Primary outcomes in young people median $\left(Q_{1} ; Q_{3}\right)$} \\
\hline \multicolumn{2}{|l|}{ Perceived health status (PedsQL 4.0 Generic Core Scale) } \\
\hline Psychosocial health & $69.2(60.0 ; 92.9)$ \\
\hline Physical health & $68.8(56.3 ; 89.1)$ \\
\hline \multicolumn{2}{|l|}{ Perceived health status (PedsQL 3.0 Rheumatology Module) } \\
\hline Treatment & $76.8(71.4 ; 100)$ \\
\hline Communication & $75.0(66.7 ; 91.7)$ \\
\hline Pain and hurt & $62.5(50 ; 93.8)$ \\
\hline Daily activities & $100.0(91.3 ; 100.0)$ \\
\hline Worry & $75.0(66.7 ; 91.7)$ \\
\hline \multicolumn{2}{|l|}{ Secondary outcomes in young people median $\left(Q_{1} ; Q_{3}\right)$} \\
\hline Global quality of life (LAS) & $73.0(68.5 ; 90.0)$ \\
\hline Illness-related knowledge (PKQ) & $31.3(18.8 ; 43.8)$ \\
\hline \multicolumn{2}{|l|}{ Fatigue (MFI-20) median (Q1;Q3) } \\
\hline Motivation & $7.0(5.3 ; 9.0)$ \\
\hline Mental fatigue & $9.5(7.0 ; 12.8)$ \\
\hline Activity & $9.5(7.0 ; 12.0)$ \\
\hline Physical fatigue & $9.5(6.3 ; 13.8)$ \\
\hline General fatigue & $9.5(7.3 ; 13.0)$ \\
\hline Presence of medication adherence (SHCS-AQ) & $23(69.7 \%)$ \\
\hline \multicolumn{2}{|l|}{ Secondary outcomes in parents median $\left(Q_{1} ; Q_{3}\right)$} \\
\hline \multicolumn{2}{|l|}{ Dimensions of parenting style median (Q1;Q3) } \\
\hline Autonomy support (PVF) & $3.8(3.3 ; 4.3)$ \\
\hline Promotion of independence (PI) & $3.4(3.1 ; 1.8)$ \\
\hline Behavioural control (PRS-YSR revised to parent self-report) & $4.0(3.6: 4.3)$ \\
\hline Psychological control (PCS-YSR revised to parent self-report) & $2.0(1.8 ; 2.5)$ \\
\hline \multicolumn{2}{|l|}{ Perceived health status (PedsQL 4.0 Generic Core Scale, parent report) } \\
\hline Psychosocial health & $85.0(71.7 ; 90.0)$ \\
\hline Physical health & $73.0(56.3 ; 96.9)$ \\
\hline \multicolumn{2}{|c|}{ Perceived health status (PedsQL 3.0 Rheumatology Module, parent report) } \\
\hline Treatment & $89.3(71.4 ; 97.3)$ \\
\hline Communication & $75.0(64.6 ; 91.7)$ \\
\hline Pain and hurt & $84.4(50.0 ; 95.3)$ \\
\hline Daily activities & $100.0(90.0 ; 100.0)$ \\
\hline Worry & $91.7(66.7 ; 100.0)$ \\
\hline
\end{tabular}


cut-off values used for the longitudinal analyses will also be used for the comparative analyses.

\section{Ethical issues}

The Institutional Review Board of the University Hospitals Leuven, Belgium, evaluated and approved the study protocol (B32220096363). This study is performed with ethical standards as described in the latest Declaration of Helsinki.

\section{Study 2: Qualitative study}

Design

We use an explanatory design to expand the quantitative results with the qualitative data. ${ }^{20}$ To gain insight into the processes underpinning the transition programme, we conduct in-depth interviews with the young people and parents of the intervention group. The specific aims of this qualitative study are (1) to get an understanding of the reasons why particular effects are observed, and (2) to evaluate the feasibility and utility of the key components of the complex intervention from the patients' and parents' perspectives.

\section{Inclusion criteria}

Those participating in the intervention group of this qualitative study (young people and their parents) are purposively sampled and invited to take part in the interviews. Sampling continues until no new themes emerge from the data (ie, until data saturation is reached).

\section{Informed consent}

Participants are included in the qualitative study after we obtain written informed consent for this particular study. If the patient is a minor, informed consent is obtained from the parents and informed assent from the patient. Anonymity is guaranteed, and patients and parents are assured that they can stop the interview at any time.

\section{Data collection}

We employ a predefined interview guide, previously adjusted by an expert panel consisting of one rheumatologist (RW), one paediatric rheumatologist (CW) and three researchers (KV, PM and $\mathrm{DH})$ in the field of care transition. We ask young people and parents to answer open-ended questions about TC, the adolescent information day, the time of transfer and general experiences about the transition programme intervention.

Young people and parents are interviewed at home, each in a different room. Different strategies are used to assess the trustworthiness of the research: investigator triangulation, bracketing and building a relationship of trust with the participant. Investigator triangulation requires that more than one investigator collect and analyse the raw data, so that the findings emerge through a consensus of a team of investigators. ${ }^{43}$ Bracketing is the process of identifying and holding in abeyance preconceived beliefs and opinions about the phenomenon under study. ${ }^{43}$ Bracketing makes the researcher aware of his or her beliefs and opinions about the phenomenon under study. To build a trusting relationship with each participant, we make certain that the interviewers are not part of the therapeutic team and that the interviews take place in a room where the participant feels comfortable. These methods ensure that researcher-related bias is minimised.

\section{Data analysis}

The interviews will be analysed by using the qualitative content analysis, according to the method of Graneheim and Lundman. ${ }^{44}$ Each interview is audiotaped and transcribed verbatim. The interviews will be read through several times to obtain a good understanding of the content. The first step in the analysis is the identification of meaning units (ie, constellations of words or statements that are related to the same central meaning). The meaning units are labelled with codes, which allow the data to be considered in new and different ways. The second step in the analysis involves sorting the codes into themes. A theme is an expression of the latent content of the text. For each theme, its content is expressed in two categories. To ensure the trustworthiness of the analysis, the authors discuss the classification of codes, categories and themes until consensus is reached.

\section{Ethical issues}

The Institutional Review Board of the University Hospitals Leuven, Belgium, evaluated and approved the study protocol. This study is performed with ethical standards as described in the latest Declaration of Helsinki.

\section{Discussion}

Previous studies have shown that the implementation of a comprehensive transition programme can have positive effects on several patient outcomes. We developed a transition programme for young people with JIA as a brief intervention. Implementation of these transition programmes in clinical practice may be more feasible, because we estimated that one full-time equivalent of manpower of the entire team could take on a case load of 250-300 transitioning patients in this transition programme. In the present article, we describe the rationale and the design of a mixed methods approach for evaluating the clinical impact of the transition programme for young people with JIA, and provide baseline data.

Our study is innovative in four aspects. First, to the best of our knowledge, this is the first evaluation of a transition programme structured as a brief intervention. Brief interventions are usually conducted in a one-on-one situation and take shorter periods of time. ${ }^{45}$ Brief interventions are predominantly used in the prevention and treatment of substance abuse, ${ }^{46}$ although they have been employed in other settings as well. ${ }^{47} 48$ Shorter time-consuming interventions are in demand, since the current trend is to implement transition 
programmes through optimal time use and cost-efficacy. However, as pointed out by McDonagh et $a t^{49}$, training of healthcare professionals will require additional resources.

Second, we designed this brief intervention as a complex intervention and used the MRC framework to perform our evaluation. It is argued that even simple interventions can be complex undertakings, mixing elements of content, intensity, duration and setting. ${ }^{45}$ Therefore, clearly specifying the steps and components of a brief intervention is needed. For this purpose, we used the criteria for the development and evaluation of complex interventions (CReDECI) in healthcare. ${ }^{19}$

Third, within this complex intervention framework, we employ a sequential mixed methods approach, more specifically an embedded experimental design. This mixed methods approach enables us to gain better insight into the clinical impact of the key components of this complex intervention. Using this approach, we integrate the findings of our different studies.

Mixed methods studies differ from multimethods studies in that the latter involve a set of monomethod studies that use different designs and that are conducted independently. Although integration is highly advocated, it is seldom conducted. The reason can be explained in two ways. ${ }^{50}$ First, researchers do not always make use of the various ways of integrating findings in their reports. They report the results of qualitative and quantitative components separately, or they report the qualitative and quantitative findings together without considering how they are related to each other. This indicates that researchers may be unaware of some of the unique insights available in mixed methods studies. In our study, we looked for convergence, divergence and discrepancy in the findings of both methods. This process is defined as crystallisation. ${ }^{50}$ Indeed, crystallisation of results and exploration of definite discrepancies can lead to further insights. Second, the integration of data and findings from different components of a mixed methods study is not always apparent in the articles emerging from them. This indicates that any unique insights available from mixed methods studies may not be disseminated in ways that are attributable to mixed methods studies.

Lastly, the results of the quantitative longitudinal and comparative studies and the qualitative studies will be separately published and will provide pilot data on the feasibility and impact of a brief transition programme on patient-reported and parent-reported outcomes in young people with JIA. The data of this forthcoming study can be used to design a randomised controlled trial testing the effectiveness of the programme in a robust way. Indeed, the effect size obtained in the present study can be used for power and sample size calculations for future studies. Admittedly, since the present study is conducted in persons with JIA, the data are not necessarily generalisable to other patient populations.
Although we use an innovative embedded experimental design in the DON'T RETARD project, some limitations should be addressed. The assessment of the patient's transition readiness is no key component of our transition programme. Recently, an increasing number of studies investigated the use of assessment tools such as the Transition Readiness Assessment Questionnaire (TRAQ) ${ }^{51}$ an instrument aiming to evaluate a set of skills and developmental tasks that should be fulfilled in order to transfer patients to adult care.

Furthermore, our brief transition programme might look more as a transfer than a transition programme. The concept of a transition programme may imply more transitional care, starting in early adolescence, whereas this brief intervention starts in the pretransfer period. Our brief intervention predominantly focuses on counselling and education rather than skills training. Still, expecting all education needs to be met in the relatively short programme described is ambitious. Moreover, regular assessments of education needs should already be initiated in paediatric care.

In addition, we have to bear in mind that the list of outcomes we use to assess the clinical impact of our programme is non-exhaustive. Additional outcomes such as self-efficacy or the level of autonomy of patients might be of interest. ${ }^{52}$

Finally, since patients who were included in the comparison group are already transferred to adult rheumatology, they are older when compared with those in the intervention group. In addition, transfer of patients in general occurred somewhat later in the era before our transition programme. This also contributes to the higher age of the comparison participants. Hence, a matching procedure on age is not possible.

\section{CONCLUSION}

This methods paper presented the rationale and design of a study intended to evaluate a transition programme for young people with JIA as a brief intervention. Here, we used the MRC framework. The embedded experimental design offers the opportunity to obtain an overall picture of the clinical impact of the transition programme on young people with JIA. Furthermore, it also allows one to get an overall picture of how these young people experience this programme. This study protocol is designed to evaluate our brief intervention. This evaluation enabled us to get more insight into the active components of our transition programme. Examination of key clinical and methodological uncertainties in the exploratory trial phase could help researchers to set up definite randomised controlled trials and guide long-term implementation.

\section{TRIAL STATUS}

The trial started 1 February 2009, and ended on 1 February 2011. 
Author affiliations

${ }^{1}$ Department of Paediatrics, University Hospitals Leuven, Leuven, Belgium ${ }^{2}$ KU Leuven Department of Public Health and Primary Care, Centre for Health Services and Nursing Research, Leuven, Belgium

${ }^{3} \mathrm{KU}$ Leuven Department of Development and Regeneration; Rheumatology, Skeletal Biology and Engineering Research Centre, University Hospitals Leuven, Leuven, Belgium

${ }^{4}$ Department of Paediatric Rheumatology, University Hospitals Leuven, Leuven, Belgium

${ }^{5}$ Research Foundation Flanders, Belgium

Acknowledgements The authors thank all participants who took part in and supported this study and this paper: the patients, parents and rheumatology healthcare providers of paediatric and adult rheumatology of the four participating centres/private practices. We also thank the administrative staff of the Department of Rheumatology of Ghent, the administrative staff of the Department of Musculoskeletal Sciences, and the administrative staff of the Department of Paediatric Rheumatology of Leuven.

Contributors PM, RW, CW and DH were responsible for the concept and design of the study, as well as analysis and interpretation of the data. $\mathrm{DH}$ and KV collected the data. DH drafted the manuscript. PM, KV, EG, RW and CW critically revised the manuscript for important intellectual content. All the authors read and approved the final manuscript.

Funding This study was supported by a scientific grant from the King Baudouin Foundation; Fonds voor Wetenschappelijk Reuma Onderzoek (fund for Scientific Rheumatism Research); and Clinical Research Fund of the University Hospitals Leuven, Belgium

\section{Competing interests None.}

Patient consent Obtained.

Ethics approval The Institutional Review Board of the University Hospital Leuven, Belgium, evaluated and approved the study protocol (B32220096363).

Provenance and peer review Not commissioned; externally peer reviewed.

Data sharing statement No additional data are available.

Open Access This is an Open Access article distributed in accordance with the Creative Commons Attribution Non Commercial (CC BY-NC 3.0) license, which permits others to distribute, remix, adapt, build upon this work noncommercially, and license their derivative works on different terms, provided the original work is properly cited and the use is non-commercial. See: http:// creativecommons.org/licenses/by-nc/3.0/

\section{REFERENCES}

1. Meleis Al. Transitions theory: middle range and situation-specific theories in nursing research and practice. New York: Springer Publishing Company, 2010.

2. American Academy of Pediatrics, American Academy of Family Physicians, American College of Physicians-American Society of Internal Medicine. A consensus statement on health care transitions for young adults with special health care needs. Pediatrics 2002;110:1304-6.

3. Cooley WC, Sagerman PJ; American Academy of Pediatrics, American Academy of Family Physicians, American College of Physicians, Transitions Clinical Report Authoring Group. Supporting the health care transition from adolescence to adulthood in the medical home. Pediatrics 2011;128:182-200.

4. Knauth A, Verstappen A, Reiss J, et al. Transition and transfer from pediatric to adult care of the young adult with complex congenital heart disease. Cardiol Clin 2006;24:619-29, vi.

5. Chaturvedi S, Jones CL, Walker RG, et al. The transition of kidney transplant recipients: a work in progress. Pediatr Nephrol 2009;24:1055-60.

6. Craig SL, Towns S, Bibby H. Moving on from paediatric to adult health care: an initial evaluation of a transition program for young people with cystic fibrosis. Int J Adolesc Med Health 2007;19:333-43.

7. Holmes-Walker DJ, Llewellyn AC, Farrell K. A transition care programme which improves diabetes control and reduces hospital admission rates in young adults with type 1 diabetes aged 15-25 years. Diabet Med 2007;24:764-9.
8. McDonagh JE, Southwood TR, Shaw KL. The impact of a coordinated transitional care programme on adolescents with juvenile idiopathic arthritis. Rheumatology (Oxford) 2007;46:161-8.

9. Remorino R, Taylor J. Smoothing things over: the transition from pediatric to adult care for kidney transplant recipients. Prog Transplant 2006;16:303-8.

10. Shaw KL, Southwood TR, McDonagh JE. Development and preliminary validation of the 'Mind the Gap' scale to assess satisfaction with transitional health care among adolescents with juvenile idiopathic arthritis. Child Care Health Dev 2007; 33:380-8.

11. Steinkamp G, Ullrich G, Muller C, et al. Transition of adult patients with cystic fibrosis from paediatric to adult care-the patients' perspective before and after start-up of an adult clinic. Eur J Med Res 2001;6:85-92.

12. Hilderson D, Westhovens R, Wouters C, et al. Transitional care for adolescents with rheumatic diseases: urgent need for more research. Child Care Health Dev 2008;34:401-2.

13. Betz CL. Transition of adolescents with special health care needs: review and analysis of the literature. Issues Compr Pediatr Nurs 2004;27:179-241

14. Hilderson D, Moons $\mathrm{P}$, Westhovens $\mathrm{R}$, et al. Attitudes of rheumatology practitioners toward transition and transfer from pediatric to adult healthcare. Rheumatol Int 2012;32:3887-96.

15. Craig P, Dieppe P, Macintyre S, et al. Developing and evaluating complex interventions: the new Medical Research Council guidance. BMJ 2008;337:a1655.

16. Campbell M, Fitzpatrick R, Haines A, et al. Framework for design and evaluation of complex interventions to improve health. BMJ 2000;321:694-6.

17. Hilderson D, Corstjens F, Moons $\mathrm{P}$, et al. Adolescents with juvenile idiopathic arthritis: who cares after the age of 16? Clin Exp Rheumatol 2010;28:790-7.

18. Chaudhry SR, Keaton M, Nasr SZ. Evaluation of a cystic fibrosis transition program from pediatric to adult care. Pediatr Pulmonol 2013;48:658-65.

19. Mohler R, Bartoszek G, Kopke S, et al. Proposed criteria for reporting the development and evaluation of complex interventions in healthcare (CReDECI): guideline development. Int J Nurs Stud 2012;49:40-6.

20. Creswell JW, Plano Clark VL. Designing and conducting mixed methods research. Thousand Oaks, CA: Sage Publications, 2007.

21. Eyckmans L, Hilderson D, Westhovens R, et al. What does it mean to grow up with juvenile idiopathic arthritis? A qualitative study on the perspectives of patients. Clin Rheumatol 2011;30:459-65.

22. Hilderson D, Eyckmans L, Van der Elst K, et al. Transfer from paediatric rheumatology to the adult rheumatology setting: experiences and expectations of young adults with juvenile idiopathic arthritis. Clin Rheumatol 2013;32:575-83.

23. Ullrich G, Mattussek S, Dressler F, et al. How do adolescents with juvenile chronic arthritis consider their disease related knowledge, their unmet service needs, and the attractiveness of various services? Eur J Med Res 2002;7:8-18.

24. Varni JW, Burwinkle TM, Seid M, et al. The PedsQL 4.0 as a pediatric population health measure: feasibility, reliability and validity. Ambul Pediatr 2003;3:329-41.

25. Varni JW, Seid M, Kurtin PS. PedsQL 4.0: reliability and validity of the Pediatric Quality of Life Inventory version 4.0 generic core scales in healthy and patient populations. Med Care 2001;39:800-12.

26. Varni JW, Seid M Smith KT, et al The PedsQ in pediatric rheumatology: reliability, validity and responsiveness of the Pediatric Quality of Life Inventory Generic Core Scales and Rheumatology Module. Arthritis Rheum 2002;46:714-25.

27. Deschamps AE, De GS, Vandamme AM, et al. Diagnostic value of different adherence measures using electronic monitoring and virologic failure as reference standards. AIDS Patient Care STDS 2008;22:735-43.

28. Hilderson D, Moons $P$, Wouters $C$, et al. Adolescents with juvenile idiopathic arthritis: what do they know about their condition? [Abstract]. Ann Rheum Dis 2011;70:756.

29. Moons P, Van Deyk K, De Bleser L, et al. Quality of life and health status in adults with congenital heart disease: a direct comparison with healthy counterparts. Eur J Cardiovasc Prev Rehabil 2006;13:407-13.

30. Lin JM, Brimmer DJ, Maloney EM, et al. Further validation of the Multidimensional Fatigue Inventory in a US adult population sample. Popul Health Metr 2009;7:18.

31. Soenens B, Vansteenkiste M, Lens W, et al. Conceptualizing parental autonomy support: adolescent perceptions of promotion of independence versus promotion of volitional functioning. Dev Psychol 2007;43:633-46. 
32. Kins E, Beyers W, Soenens B, et al. Patterns of home leaving and subjective well-being in emerging adulthood: the role of motivational processes and parental autonomy support. Dev Psychol 2009;45:1416-29.

33. Soenens B, Vansteenkiste M, Luyckx K, et al. Parenting and adolescent problem behavior: an integrated model with adolescent self-disclosure and perceived parental knowledge as intervening variables. Dev Psychol 2006;42:305-18.

34. Eddy L, Cruz M. The relationship between fatigue and quality of life in children with chronic health problems: a systematic review. J Spec Pediatr Nurs 2007;12:105-14.

35. Russo E, Trevisi E, Zulian F, et al. Psychological profile in children and adolescents with severe course juvenile idiopathic arthritis. ScientificWorldJournal 2012;2012:841375

36. Wallace CA, Ruperto N, Giannini E; Childhood Arthritis and Rheumatology Research Alliance; Pediatric Rheumatology International Trials Organization; Pediatric Rheumatology Collaborative Study Group. Preliminary criteria for clinical remission for select categories of juvenile idiopathic arthritis. J Rheumatol 2004;31:2290-4.

37. Ouwerkerk JW, van Pelt PA, Takken T, et al. Evaluating score distributions in the revised Dutch version of the Childhood Health Assessment Questionnaire. Pediatr Rheumatol Online J 2008;6:14.

38. Singh G, Athreya BH, Fries JF, et al. Measurement of health status in children with juvenile rheumatoid arthritis. Arthritis Rheum 1994;37:1761-9.

39. Takken $\mathrm{T}$, van den Eijkhof $\mathrm{F}$, Hoijtink $\mathrm{H}$, et al. Examining the psychometric characteristics of the Dutch childhood health assessment questionnaire: room for improvement? Rheumatol Int 2006;26:979-83.

40. Cohen J. A power primer. Psychol Bull 1992;112:155-9.

41. Berben L, Sereika SM, Engberg S. Effect size estimation: methods and examples. Int J Nurs Stud 2012;49:1039-47.

42. Cohen J. Statistical power analysis for the behavioral sciences. Lawrence Erlbaum Associates, 1988.
43. Polit DF, Beck CT. Nursing research: principles and methods. In nursing research: principle and methods. 7 th edn. Philadelphia, PA: Lippincott Williams \& Wilkins, 2004.

44. Graneheim UH, Lundman B. Qualitative content analysis in nursing research: concepts, procedures and measures to achieve trustworthiness. Nurse Educ Today 2004;24:105-12.

45. Babor TF. Avoiding the horrid and beastly sin of drunkenness: does dissuasion make a difference? J Consult Clin Psychol 1994;62:1127-40.

46. Humeniuk $\mathrm{R}$, Ali $\mathrm{R}$, Babor $\mathrm{T}$, et al. A randomized controlled trial of a brief intervention for illicit drugs linked to the Alcohol, Smoking and Substance Involvement Screening Test (ASSIST) in clients recruited from primary health-care settings in four countries. Addiction 2012;107:957-66.

47. Fachini A, Aliane PP, Martinez EZ, et al. Efficacy of brief alcohol screening intervention for college students (BASICS): a meta-analysis of randomized controlled trials. Subst Abuse Treat Prev Policy 2012;7:40.

48. Laure $P$, Mangin $G$. Advising parents on physical activity for children between 0 and 5 years. J Sports Med Phys Fitness 2011:51:467-72.

49. McDonagh JE, Southwood TR, Shaw KL; Bristish Paediatric Rheumatology Group: Unmet education and training needs of rheumatology health professionals in adolescent health and transitional care. Rheumatology (Oxford) 2004;43:737-43.

50. O'Cathain A, Murphy E, Nicholl J. Integration and publications as indicators of 'yield' from mixed methods studies. J Mix Methods Res 2007;1:147-63.

51. Sawicki GS, Lukens-Bull K, Yin X, et al. Measuring the transition readiness of youth with special healthcare needs: validation of the TRAQ_-TransitionReadiness Assessment Questionnaire. J Pediatr Psychol 2011;36:160-71.

52. van Staa A, van der Stege HA, Jedeloo S, et al. Readiness to transfer to adult care of adolescents with chronic conditions: exploration of associated factors. J Adolesc Health 2011;48:295-302 